DIW BERLIN

Discussion Papers

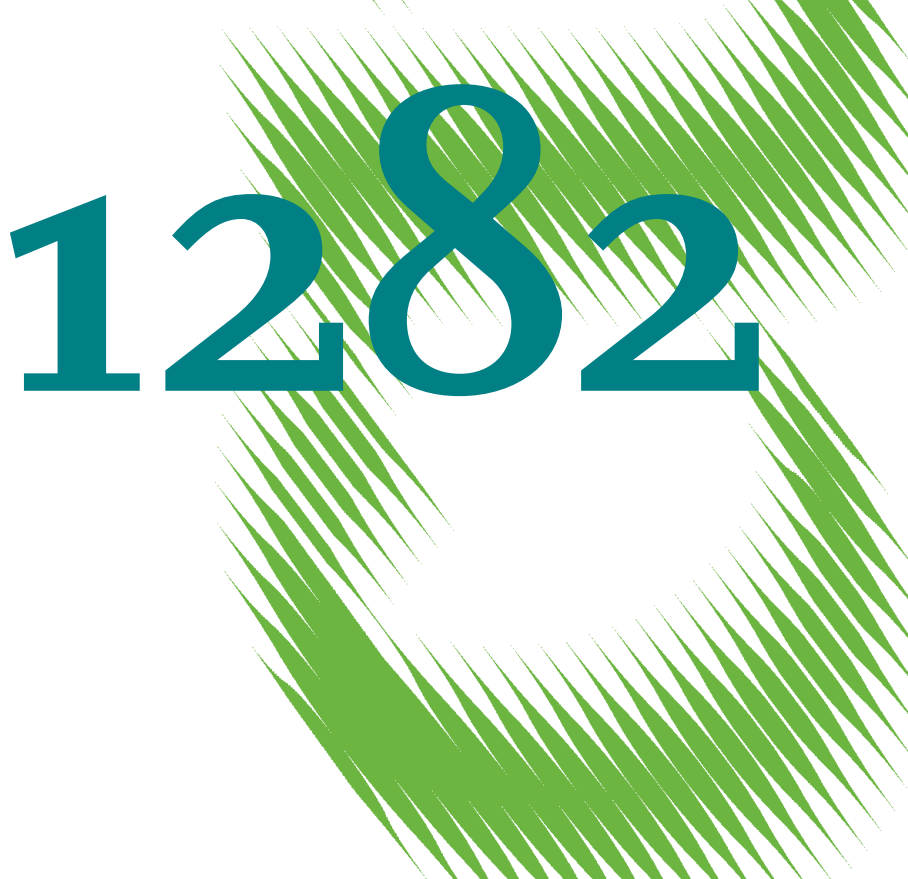

Forecasting the Risk of Speculative Assets by Means of Copula Distributions 
Opinions expressed in this paper are those of the author(s) and do not necessarily reflect views of the institute.

IMPRESSUM

(C) DIW Berlin, 2013

DIW Berlin

German Institute for Economic Research

Mohrenstr. 58

10117 Berlin

Tel. $+49(30) 89789-0$

Fax +49 (30) $89789-200$

http://www.diw.de

ISSN print edition $1433-0210$

ISSN electronic edition 1619-4535

Papers can be downloaded free of charge from the DIW Berlin website:

http://www.diw.de/discussionpapers

Discussion Papers of DIW Berlin are indexed in RePEc and SSRN:

http://ideas.repec.org/s/diw/diwwpp.html

http://www.ssrn.com/link/DIW-Berlin-German-Inst-Econ-Res.html 


\title{
Forecasting the risk of speculative assets by means of copula distributions
}

\author{
Benjamin Beckers*, Helmut Herwartz ${ }^{\dagger}$ and Moritz Seidel ${ }^{\ddagger}$
}

March 19, 2013

\begin{abstract}
The $\operatorname{GARCH}(1,1)$ model and its extensions have become a standard econometric tool for modeling volatility dynamics of financial returns and portfolio risk. In this paper, we propose an adjustment of GARCH implied conditional value-at-risk and expected shortfall forecasts that exploits the predictive content of uncorrelated, yet dependent model innovations. The adjustment is motivated by non-Gaussian characteristics of model residuals, and is implemented in a semiparametric fashion by means of conditional moments of simulated bivariate standardized copula distributions. We conduct in-sample forecasting comparisons for a set of 18 stock market indices. In total, four competing copula-GARCH models are contrasted against each other on the basis of their one-step ahead forecasting performance. With regard to forecast unbiasedness and precision, especially the Frank-GARCH models provide most conservative risk forecasts and outperform all rival models.
\end{abstract}

Keywords: copula distributions, expected shortfall, GARCH, model selection, non-Gaussian innovations, risk forecasting, value-at-risk.

JEL Classification: C22; C51; C52; C53; G32

\footnotetext{
*DIW Berlin, Graduate Center, Mohrenstraße 58, 10117 Berlin, Germany, bbeckers@diw.de.

${ }^{\dagger}$ Chair of Econometrics, University of Göttingen, Platz der Göttinger Sieben 5, 37037 Göttingen, Germany, hherwartz@uni-goettingen.de.

${ }_{\ddagger}^{\ddagger}$ Deutsche Bundesbank, Wilhelm-Epstein-Straße 14, 60431 Frankfurt am Main, Germany, moritz.seidel@bundesbank.de. This paper was completed while Moritz Seidel was at the University of Kiel. The views expressed herein are those of the authors and should not be attributed to the Deutsche Bundesbank.
} 



\section{Introduction}

In 1996 the Basel Committee for Banking Supervision stipulated the calculation of a $1 \%$ value-at-risk (VaR) as the standard method for measuring market risk in financial institutions (Basel Committee on Banking Supervision, 2005). Moreover, to prevent financial institutions from underestimating market risks, the Committee defined rules on backtesting methods for internal risk models and implemented a system of multiplication factors for penalizing those models that fail the backtesting criteria. Despite its prominent use in risk management, VaR has been critized for several drawbacks. Owing to its failure in capturing the entire tail risk beyond the nominal level, its procyclicality and the incentives it provides for financial institutions to take on tail risks, the Basel Committee may favor the expected shortfall (ES) as the new standard risk measure in the future (Basel Committee on Banking Supervision, 2012). In particular, ES refines tail risk evaluation. To cover a larger range of extreme events it is further discussed to raise the nominal $1 \%$ benchmark risk level to some less conservative threshold.

In financial practice and particularly in portfolio risk management, GARCH models have become a standard econometric tool for modeling the characteristic volatility dynamics of financial returns and for ex-ante evaluation of risk measures such as VaR and ES (see Angelidis \& Degiannakis (2007) for an overview of GARCH VaR- and McNeil \& Frey (2000) and Zhu \& Galbraith (2011) for two examples for GARCH ES forecast models). Since their introduction by Engle (1982) and Bollerslev (1986), various GARCH extensions accounting for asymmetries and/or non-linear behavior in the volatility process have been made, and generally improved the understanding of the second order dynamics of financial time series. Apart from specification issues, moreover, the assumption of conditional normality in the models' uncorrelated standardized residuals has been questioned. As a consequence, fat-tailed, non-normal conditional distributions have been proposed to improve both the GARCH implied approximation of em-

pirical returns (e.g. Bollerslev (1990)) and forecasts of volatility and other risk measures (see Zhu \& Galbraith (2011) for an overview on this literature). None of these models incorporating non-normal disturbance distributions, however, have considered the standardized residuals to exhibit a dependence structure beyond zero linear correlation that can be exploited for forecasting purposes. If subsequent residuals extracted from estimated univariate GARCH processes - despite 
being uncorrelated - are dependent in higher moments, they may carry predictive content that could be exploited to improve VaR and ES forecasts. As proposed in Herwartz (2012), the standardized copula distributions introduced by Lee \& Long (2009) can be employed to model the dependence structure in GARCH innovations in a flexible manner.

Against this background, this study translates the work of Lee \& Long (2009) to the univariate setting and closely follows Herwartz (2012) who advocates copula-autoregressive models for the forecasting of macroeconomic data. The aim of this study is to investigate the predictive content of uncorrelated yet possibly autodependent, consecutive GARCH model residuals by means of (standardized) copula distributions in order to improve risk forecasts of models assuming Gaussian or iid innovations. In particular, a copula that best describes GARCH implied model innovations shall be selected. After estimating its conditional tail characteristics, the model implied (conditional) VaR and ES predictions will be cast against their standard univariate Gaussian-GARCH counterpart. These forecasts are evaluated for a set of 18 stock market indices. The in-sample forecasting performance is assessed for four competing families of copula-GARCH models, the Clayton, Gumbel and Frank copula and the Gaussian copula for the purpose of benchmarking the empirical results. To allow a flexible matching of residual sequences with copula innovations, alternative parameterizations and rotations are considered for each Archimedean copula family.

To preview some results, we find that consecutive standardized threshold GARCH (TGARCH) innovations exhibit a dependence structure that differs from both conditional normality or independence, and can generally be best described by the standardized Frank copula distribution. Based on the standardized Frank copula, VaR and ES predictions are more conservative and outperform risk forecasts from standard Gaussian-TGARCH models. Assuming a simple economic loss function of a regulator, it is shown that losses can be markedly reduced when relying on risk forecasts from Frank-TGARCH models.

The next section outlines the copula-TGARCH model. Subsequently, Section 3 describes the VaR and ES forecasting in the framework of the proposed model and the applied backtesting procedures. Section 4 provides empirical results. Section 5 concludes. Issues of evaluating standardized copula distributions by means of simulations and the data driven selection of candidate copula models 
are described in the Appendix.

\section{The copula-TGARCH model}

In this section we first briefly introduce the TGARCH model to formalize time variation in second order return properties. Then, with some empirical estimates at hand, we will argue that TGARCH innovations show nontrivial higher order dependence. Thirdly, we propose a semiparametric model class that allows the embedding of such complicated dependence patterns.

\subsection{The TGARCH model}

The TGARCH(1,1,1) ${ }^{1}$ model introduced by Glosten et al. (1993) accounts for asymmetries in the volatility response. Conditional on the information available at time $t-1$, denoted $\Omega_{t-1}$, first and second order return characteristics are given as

$$
\begin{aligned}
r_{t} & =\mu_{t}+\sigma_{t} \xi_{t} \\
\sigma_{t}^{2} & =\gamma_{0}+\gamma_{1}\left(r_{t-1}-\mu_{t-1}\right)^{2}+\gamma_{1}^{-}\left(r_{t-1}-\mu_{t-1}\right)^{2} I\left(r_{t-1}-\mu_{t-1}<0\right)+\beta_{1} \sigma_{t-1}^{2}
\end{aligned}
$$

where $I()$ is an indicator function. Moreover, $\sigma_{t}^{2}$ is the time-dependent variance of the daily log-return sequence $r_{t}, \mu_{t}$ the conditional mean return and $\gamma_{0}, \gamma_{1}, \gamma_{1}^{-}$ and $\beta_{1}$ denote the variance response parameters. Noting that we will empirically analyse daily returns, we set $\mu_{t}=0$ and, thus, $r_{t}=\sigma_{t} \xi_{t}$. The TGARCH-model innovations $\xi_{t}=r_{t} / \sigma_{t}$ are typically assumed to follow an iid Gaussian distribution. As an illustration of potential higher-order dependence, Figure 1 displays scatter diagrams of model estimates $\hat{\xi}_{t}$ and $\hat{\xi}_{t+1}$ for four stock market indices. It can be seen that the assumptions of independence and a joint normal distribution are questionable. Instead there seems to prevail a remaining dependence structure characterized by a clustering of subsequently negative (positive for NIKKEI) innovations. Hence, empirical TGARCH residuals are characterized by joint lower

\footnotetext{
${ }^{1}$ For space considerations we focus on the TGARCH $(1,1,1)$ model. For the GARCH(1,1) specification we generally find slightly stronger dependence patterns in model innovations. Qualitatively, the empirical performance of the copula-GARCH models is in full analogy to that of copula-TGARCH specifications that will be discussed in this work.
} 
(upper) tail dependence.

With the diagnosis of higher order dependence at hand, one may ask for a model framework suitable to exploit such autodependence patterns for practical issues of return or return quantile prediction. While the dependence in higher order moments might be easily diagnosed by means of tests for nonlinearity, the respective test approaches are often not decisive in how to arrive at (parametric) conditional ex-ante forecasts. To allow for a flexible and data driven evaluation of nonlinear relationships we propose a semiparametric model class advocated in Herwartz (2012) that comprises standardized copula distributions. As we argue below, this model class allows to evaluate the conditional characteristics of TGARCH innovations $\xi_{t+1}$ given $\xi_{t}$. In consequence, even with Gaussian marginals, $\xi_{t} \sim N(0,1)$, the proposed model framework allows for nontrivial VaR and ES forecasts.

\subsection{Standardized copula implied dependence structures}

A priori the space of potential autodependence patterns appears overly large to define a rich parametric model class that may nest most promising nonlinear model approaches. Instead, one may opt for a semiparametric device that allows to flexibly translate empirical dependence structures into the two dimensional space of $\left(\xi_{t}, \xi_{t+1}\right)$. The standardized copulae as introduced by Lee \& Long (2009) provide a framework generating a rich dependence structure.

Below we follow Herwartz $(2012)$ in adopting the approach of Lee \& Long (2009). First, a vector $\mathbf{v}_{t}=\left(\xi_{t}, \xi_{t+1}\right)^{\prime}$ of standardized innovations is defined which is drawn from a standardized bivariate copula distribution, as

$$
\mathbf{v}_{t}=\Sigma^{-\frac{1}{2}} \mathbf{w}_{t}^{(1)}, \quad \mathbf{w}_{t}^{(1)} \sim \mathcal{C}_{\delta}\left(\Phi\left(w_{1, t}\right), \Phi\left(w_{2, t}\right)\right), \quad \mathrm{E}\left[\mathbf{w}_{t}^{(1)}\right]=0, \quad \operatorname{Cov}\left[\mathbf{w}_{t}^{(1)}\right]=\Sigma
$$

where $\Phi($.$) and \mathcal{C}_{\delta}($.$) denote the Gaussian distribution function and a copula$ function with parameter $\delta$, respectively $\left.\right|^{2}$ Standardization is crucial, since the elements of the random vectors $\mathbf{w}_{t}^{(1)}$ exhibit an a priori specified correlation, e.g. measured by means of Kendall's $\tau$. This also implies a covariance structure deviating from the identity matrix $\mathbf{I}$. To be precise we set, $\Sigma^{1 / 2}=\Gamma \Lambda^{1 / 2} \Gamma^{\prime}$, where $\Lambda$ is

${ }^{2}$ See Cherubini et al. (2004) or Nelsen $(2006)$ for textbook treatments of copulae. 
a diagonal matrix collecting the eigenvalues of $\Sigma$ and $\Gamma$ is the matrix of respective eigenvectors. Formally, the assumptions on $\mathbf{w}_{t}^{(1)}$ imply $\mathrm{E}\left[\mathbf{v}_{t}\right]=0$ and $\mathrm{E}\left[\mathbf{v}_{t} \mathbf{v}_{t}^{\prime}\right]=\mathbf{I}$. Hence, standardization assures that the elements in TGARCH innovation tuples $\left(\xi_{t}, \xi_{t+1}\right)^{\prime}$ remain uncorrelated but the model now allows for non-normality and for a higher order dependence structure (Lee \& Long, 2009). Presuming Gaussian marginals the diagonal elements of $\Sigma$ are unity, whereas the off-diagonal elements of $\Sigma$ are determined by the (sample) covariance of the elements in $\mathbf{w}_{t}^{(1)}$. The model also covers joint normality and independence of $\xi_{t}$ and $\xi_{t+1}$ if $\mathcal{C}($.$) is$ the Gaussian copula. While the unconditional first and second order moments of elements in $\mathbf{v}_{t}$ are identical over alternative copulae, their conditional moments $E\left[\xi_{t+1} \mid \xi_{t}\right]$ are model-specific, since each copula is characterized by a distinct CDF and hence implies model-specific conditional quantiles (Herwartz, 2012).

The set of copulae employed in this study includes the three Archimedean families Clayton $\left(\mathcal{C}_{\delta}^{C}\right)$, Gumbel $\left(\mathcal{C}_{\delta}^{G}\right)$ and Frank $\left(\mathcal{C}_{\delta}^{F}\right)$ and the Gaussian copula $\left(\mathcal{C}^{\Phi}\right)$. With arguments $u_{1}$ and $u_{2}$ the former read, respectively, as

$$
\begin{aligned}
& \mathcal{C}_{\delta}^{C}\left(u_{1}, u_{2}\right)=\max \left[\left(u_{1}^{-\delta}+u_{2}^{-\delta}-1\right)^{-\frac{1}{\delta}}, 0\right] \\
& \mathcal{C}_{\delta}^{G}\left(u_{1}, u_{2}\right)=\exp \left[-\left[\left(-\ln \left(u_{1}\right)\right)^{\delta}+\left(-\ln \left(u_{2}\right)\right)^{\delta}\right]^{-\frac{1}{\delta}}\right] \text { and } \\
& \mathcal{C}_{\delta}^{F}\left(u_{1}, u_{2}\right)=-\frac{1}{\delta} \ln \left(1+\frac{\left(\exp \left(-\delta u_{1}\right)-1\right)\left(\exp \left(-\delta u_{2}\right)-1\right)}{\exp (-\delta)-1}\right) .
\end{aligned}
$$

Figure 2 illustrates by means of scatter diagrams the distributional form of the standardized bivariate Gaussian and Archimedean copulae with an identical dependence parameter $\tau=0.25$ prior to standardization. Comparing the scatter diagrams from the Archimedean copulae with the empirical distributions of TGARCH innovations (Figure 1) motivates their use in exploiting the higher order dependence structure of empirical TGARCH innovations for forecast adjustments. Notice that the Clayton and Gumbel copula feature lower and upper tail dependence, respectively. Figure 1, however, indicates that lower tail dependence might be more characteristic for empirical TGARCH innovations. Therefore, in addition to the specifications in (3) to (6), we consider three rotations of the Clayton and Gumbel copula and one of the radially symmetric Frank copula. 
Random variables from rotated copulae are obtained as

$$
\begin{aligned}
\mathbf{w}_{t}^{(2)} & \sim \mathcal{C}_{\delta}^{\bullet}\left(1-\Phi\left(w_{1, t}\right), \Phi\left(w_{2, t}\right)\right), \\
\mathbf{w}_{t}^{(3)} & \sim \mathcal{C}_{\delta}^{\bullet}\left(\Phi\left(w_{1, t}\right), 1-\Phi\left(w_{2, t}\right)\right) \text { and } \\
\mathbf{w}_{t}^{(4)} & \sim \mathcal{C}_{\delta}^{\bullet}\left(1-\Phi\left(w_{1, t}\right), 1-\Phi\left(w_{2, t}\right)\right),
\end{aligned}
$$

where $\bullet\{C, G, F\}$. With regard to fitting rotated copula innovations to empirical residuals, it can consequently be argued that rotations 2 and 4 of Gumbel, and similarly rotations 1 and 3 of Clayton might provide closest approximations of real data, since these specifications are characterized by lower tail dependence. Moreover, as illustrated in Figure 3 for medium dependence parameters, the rotated symmetric Frank copula is characterized by slightly more mass in the joint lower and upper quantiles, and, hence, might be expected to provide better approximations of empirical TGARCH innovations than its unrotated counterpart.

To improve the flexibility in matching residual sequences with copula innovations, we consider a set of parameters $\delta$ for each (rotated) copula. Here $\delta$ is chosen such that, prior to standardization, for each family a set of copulae is obtained that is uniquely characterized by a desired dependence level (measured by Kendall's $\tau$ ). The target values for $\tau$ are for Clayton, Gumbel and Frank ${ }^{3}$ respectively, $\tau^{C, G}=\{0.010,0.015, \ldots, 0.075,0.100,0.150, \ldots, 0.250\}$ and $\tau^{F}=\{0.010,0.025,0.050, \ldots, 0.100,0.150, \ldots, 0.750\}$.

\section{Copula adjusted risk forecasts}

This section first describes risk forecasting by means of copula-adjusted TGARCH models. Then, the criteria for risk forecast assessment are provided. The implementation of the semiparametric forecast adjustment, i.e. copula selection and the estimation of conditional tail distributions is discussed in Appendix A in detail.

\footnotetext{
${ }^{3}$ For the relation between $\delta$ and $\tau$ of the three copula families, see Cherubini et al. $(2004$, Table 3.5). For the Clayton and Gumbel copulae, larger target values up to $\tau=0.75$ were also tried but provided poorer fits to empirical data when applying the routine outlined in Appendix A.1.
} 


\subsection{Conditional VaR and ES forecasts}

Conditional on the information set $\Omega_{t}$ the $\mathrm{VaR}(\mathrm{cVaR})$ at level $\alpha$ is the (negative) quantile $q_{\alpha}$ for which the probability of the one period-ahead return being less than $q_{\alpha}$ is no larger than $\alpha$. In this work we distinguish nominal coverage levels $\alpha=(0.01,0.025,0.05)^{\prime}$ for empirical forecast assessments. Formally, the cVaR reads as

$$
\operatorname{cVaR}_{\alpha}\left(r_{t+1} \mid \Omega_{t}\right)=-q_{\alpha}\left(r_{t+1} \mid \Omega_{t}\right)=-F^{-1}\left(\alpha \mid \Omega_{t}\right),
$$

where $F^{-1}\left(\alpha \mid \Omega_{t}\right)$ denotes the inverse of the conditional CDF (cCDF) of returns $r_{t+1}$. Related to the VaR, ES is the expected loss, given that this loss exceeds the VaR. Formally, the conditional ES (cES) of $r_{t+1}$ is

$$
\operatorname{cES}_{\alpha}\left(r_{t+1} \mid \Omega_{t}\right)=-\mathrm{E}\left[r_{t+1} \mid\left(r_{t+1}<-\mathrm{cVaR}_{\alpha}\left(r_{t+1} \mid \Omega_{t}\right)\right)\right] .
$$

By construction, the $\mathrm{cES}$ is more conservative than the cVaR since $\mathrm{cES} \mathrm{S}_{\alpha}\left(r_{t+1}\right) \geq$ $\mathrm{cVaR}_{\alpha}\left(r_{t+1}\right)$.

Determining either the $\mathrm{cVaR}$ or the $\mathrm{cES}$ requires an estimate of the $\mathrm{cCDF}$ of returns $F\left(r_{t+1} \mid \Omega_{t}\right)$. Since $r_{t+1}=\sigma_{t+1} \xi_{t+1}$, this estimate depends on both, the (one-period ahead) prediction of the conditional variance $\sigma_{t+1}$ and on the cCDF of the innovation sequence $F\left(\xi_{t+1} \mid \xi_{t}\right)$. While a one-period ahead volatility forecast for the TGARCH $(1,1,1)$ can be obtained immediately from (2) by means of QML parameter estimates,

$$
\hat{\sigma}_{t+1}^{2}=\hat{\gamma}_{0}+\hat{\gamma}_{1} r_{t}^{2}+\hat{\gamma}_{1}^{-} r_{t}^{2} I\left(r_{t}<0\right)+\hat{\beta}_{1} \hat{\sigma}_{t}^{2},
$$

the $\mathrm{cCDF}$ of the innovation sequence depends on the underlying copula distribution. Hence, for both risk forecasts, it is crucial to obtain an estimate of $F_{\delta}^{\bullet}\left[\xi_{t+1} \mid \xi_{t}\right]$ for each (rotated) copula $\bullet \in\{\Phi, C, F, G\}$ and parameter $\delta$. In the Gaussian model, $\bullet=\Phi$, this is trivial due to independence of the model innovations. Under higher order dependence, however, the conditional distribution involves a complex expression of mixed marginal distributions with a copula specific dependence structure. Therefore an analytical solution is hardly feasible. As an alternative, one can rely on simulated innovations and estimate the cCDF for each parameterized copula (Herwartz, 2012). 
With the cCDF of the innovations at hand, quantiles and expected quantile "loss" for the distribution of $\xi_{t+1}$ given $\xi_{t}$ can be obtained in a similar fashion to (10) and (11). The copula-adjusted cVaR and cES forecasts for returns $r_{t+1}$ read as

$$
\begin{aligned}
\operatorname{cVaR}_{\alpha}^{\bullet}\left(r_{t+1} \mid \Omega_{t}\right) & =-\hat{\sigma}_{t+1} q_{\alpha}^{\bullet}\left(\xi_{t+1} \mid \xi_{t}\right) \text { and } \\
\operatorname{cES}_{\alpha}^{\bullet}\left(r_{t+1} \mid \Omega_{t}\right) & =-\hat{\sigma}_{t+1} \mathrm{E}\left[\xi_{t+1} \mid\left(\xi_{t+1}<q_{\alpha}^{\bullet}\left(\xi_{t+1} \mid \xi_{t}\right)\right)\right]
\end{aligned}
$$

respectively. The copula-based adjustment of $\mathrm{cVaR}$ and $\mathrm{cES}$ forecasts has to be derived from the unknown conditional distribution of $\xi_{t+1}$ given $\xi_{t}$. To allow for sufficient flexibility in describing this distribution, the model space in this study includes a large set of differently parametrized and rotated copulae (see Section 2.2. The approach to select the copula that best describes the distribution of empirical standardized TGARCH innovations and to estimate its tail distribution are detailed in Appendices A.1 and A.2, respectively.

\subsection{Forecast evaluation}

While the evaluation of VaR models has seen numerous applications, tests on ES forecast accuracy have been conducted only recently (see McNeil \& Frey (2000), Angelidis \& Degiannakis (2007), Diks et al. (2011) and Zhu \& Galbraith (2011)). The competing copula-specific cVaR and cES forecasts obtained by 13 and (14) can be compared with regard to unbiasedness and accuracy to discriminate against misspecified models.

Generally, the backtesting of VaR forecasts relies on the series of so-called VaR hits, i.e.

$$
h i t_{t+1, \alpha}^{\bullet}=I\left(r_{t+1} \leq-\widehat{\mathrm{cVaR}}_{\alpha}^{\bullet}\left(r_{t+1} \mid \Omega_{t}\right)\right)
$$

Since the cES is only defined for $r_{t+1} \leq-\widehat{\mathrm{CVaR}}_{\alpha}^{\bullet}\left(r_{t+1} \mid \Omega_{t}\right)$, a correct specification of the hit series is required prior to cES forecast evaluation. Thus, in principle tests on cVaR forecasts can be carried out based on the entire length of the hit sequence, while cES tests potentially suffer from data scarcity since they depend on the condition of observing a cVaR hit first ${ }^{4}$ For this reason, we put

\footnotetext{
${ }^{4}$ To deal with this problem Zhu \& Galbraith (2011) define a hit as a return below a certain
} 
particular emphasis on the evaluation of cES forecasts by means of both common statistical loss functions and a more economic criterion that is motivated from the perspective of a representative regulator. Next, we outline a prominent VaR diagnostic and turn to alternative tools for cES evaluation.

\subsubsection{Value-at-risk diagnosis}

For the evaluation of cVaR estimates we apply the dynamic quantile test by Engle \& Manganelli (2004) formalizing a correct unconditional and conditional VaR specification under the null hypothesis. Let '•' indicate a particular model employed for risk assessment. In this framework it is tested, if the centered hit sequence $\widetilde{h i t}_{t+1, \alpha}^{\bullet}=h i t_{t+1, \alpha}^{\bullet}-\alpha$ follows a martingale difference sequence. We restrict our analysis to the following regression model including five lags of centered hits

$$
\widetilde{h i t}_{t+1, \alpha}^{\bullet}=\beta_{0}+\sum_{k=1}^{5} \beta_{k} \widetilde{h i t}_{t-k+1, \alpha}^{\bullet}+u_{t+1}
$$

The null hypothesis of correct conditional and unconditional coverage of the model, hence, reads as $H_{0}: \beta_{k}=0, \quad \forall k=0, \ldots, 5$.

\subsubsection{Expected shortfall diagnosis}

We employ the following criteria to evaluate cES forecasts:

- Standardized cES errors

McNeil \& Frey (2000) suggest to assess the correct specification of cES forecasts by means of standardized cES forecast errors

$$
\hat{e}_{t+1}=\frac{r_{t+1}-\left(-\widehat{\mathrm{cES}}_{\alpha}^{\bullet}\left(r_{t+1} \mid \Omega_{t}\right)\right)}{\hat{\sigma}_{t+1}}, \forall r_{t+1} \leq-\widehat{\mathrm{cVaR}}_{\alpha}^{\bullet}\left(r_{t+1} \mid \Omega_{t}\right)
$$

where $\hat{\sigma}_{t+1}$ is the GARCH standard deviation forecast. If the model is correctly specified, the exceedance residuals $\hat{e}_{t+1}$ should be iid with mean zero. We follow the approach in Diks et al. (2011) and conduct a two-sided $t$-test of the null hypothesis $H_{0}: E\left[e_{t}\right]=0$ with a HAC variance estimator.

threshold loss of $-1.2 \%$ to $-0.6 \%$ and thereby differentiate the cES forecast evaluation from the cVaR diagnosis. These threshold levels are, however, not sufficiently conservative for practical applications. 
- Mean-squared error

Similar to Angelidis \& Degiannakis (2007) and Zhu \& Galbraith (2011), we also employ the mean squared error (MSE) to compare overall cES forecasting accuracy. The MSE is defined as

$$
M S E_{\alpha}^{\bullet}=\frac{1}{\tilde{T}^{\bullet}} \sum_{t=1}^{T} l_{t+1, \alpha}^{2, \bullet},
$$

with $\tilde{T}^{\bullet}$ denoting the model-specific number of $\mathrm{cVaR}$ hits and

$$
l_{t+1, \alpha}^{2, \bullet}= \begin{cases}\left(\left|r_{t+1}\right|-\widehat{\mathrm{cES}}_{\alpha}^{\bullet}\left(r_{t+1} \mid \Omega_{t}\right)\right)^{2} & \text { if } r_{t+1} \leq-\widehat{\mathrm{cVaR}}_{\alpha}^{\bullet}\left(r_{t+1} \mid \Omega_{t}\right) \\ 0 & \text { otherwise }\end{cases}
$$

- Regulator's loss

As Granger \& Pesaran (2000) point out, the choice of a loss functional should reflect the objectives of the forecast user. Therefore, we follow Sarma et al. (2003) and consider additionally a potential loss function of a regulating institution. Employing the ES as a regulation criterion, a regulator's loss may read as

$$
R L_{\alpha}^{\bullet}=\frac{1}{\breve{T} \bullet} \sum_{t=1}^{T} l_{t+1, \alpha}^{r, \bullet}
$$

with $\breve{T} \bullet$ denoting the model-specific number of cES hits, and

$$
l_{t+1, \alpha}^{r, \bullet}= \begin{cases}\left(\left|r_{t+1}\right|-\widehat{\mathrm{cES}}_{\alpha}^{\bullet}\left(r_{t+1} \mid \Omega_{t}\right)\right)^{2} & \text { if } r_{t+1} \leq-\widehat{\mathrm{cES}}_{\alpha}^{\bullet}\left(r_{t+1} \mid \Omega_{t}\right) \\ 0 & \text { otherwise. }\end{cases}
$$

The intuition behind this loss function is that the regulating authority is mainly interested in negative ES shocks, i.e. situations where a loss exceeds the predicted cES.

In order to test if two models differ significantly in their forecasting accuracy we employ the finite-sample sign test as proposed by Diebold \& Mariano (1995) 5

\footnotetext{
${ }^{5}$ The asymptotic Diebold-Mariano test (Diebold \& Mariano (1995)) is hardly applicable in our setting for two reasons. First, it is generally not suitable for the comparison of cES forecasts due to the rare occurrence of hits. Second, since the cES is not defined in most time-periods, autocovariances at different time lags are ill defined. A potentially more powerful finite-sample
} 
The test refers to the null hypothesis that two competing forecasts do not differ in terms of accuracy. For this purpose, the loss differential $d_{t}=l_{t}^{\bullet}-l_{t}^{\triangleright}$ between competing models, indicated by $\bullet$ and $\diamond$, is constructed and the null hypothesis reads as $H_{0}: \operatorname{median}\left(d_{t}\right)=0$. For the application of the sign test in our setting, it is decisive to specify the set of hits under consideration as these usually differ across competing cES forecasts $\widehat{\mathrm{cES}}_{\alpha}^{\bullet}$ and $\widehat{\mathrm{cES}}_{\alpha}^{\diamond}$. Here, we employ the union set of hits and, thus, consider all periods when returns hit the least conservative cVaR (for $M S E_{\alpha}^{\bullet}$ ) or cES (for $R L_{\alpha}^{\bullet}$ ) forecast. Then, in case that only one model features a hit as defined in (18) or (20), this model's forecast loss will be positive, while the other forecast loss is zero. The loss differential will, hence, indicate a superiority of the model that has not been hit. If two forecasts generally differ in their level (implying possibly distinct unconditional VaR-coverage rates), such a choice will implicitly punish the less conservative cVaR or cES forecast for the larger number of hits. This is particularly important for the MSE criterion, as this punishment partially offsets a bias resulting from the specification of forecast errors. In detail, if the period loss exceeds both cVaR forecasts but not the less conservative cES forecast, the squared error would be larger for the more conservative cES forecast by construction (see (18)). Since most losses left of the cVaR forecast can be expected to be close to this forecast value rather than deep in the tail, a more conservative cES may obtain a larger MSE than a less conservative cES. For regulator's losses this is irrelevant, since the more conservative forecast always yields a smaller loss by construction.

\section{Empirical results}

In this section we discuss the copula-specific cVaR and cES forecasting performance. In the first place, however, we introduce the data and discuss a few results on the in-sample features of the estimated TGARCH innovations and the subsequent copula selection outlined in Appendix A.1.

alternative to the sign test is Wilcoxon's signed-rank test which partially accounts for the size of the loss differential. However, the symmetry condition required for the loss differential distribution is found to be violated here. 


\subsection{Data}

We use financial data from a set of 18 market indices to analyze the cVaR and cES forecasting performance of selected copula-TGARCH models. For these indices about 2300 daily observations cover the time period from 1 May 2003 until 30 April 2012.6 $!^{6}$ The set of indices and respective parameter estimates for the TGARCH $(1,1,1)$ model are shown in Table 1. Significant leverage effects $\left(\gamma_{1}^{-}>0\right)$ can be diagnosed for all markets.

\subsection{Copula selection}

As motivated in Section 2.2, the applied matching procedure finds strong evidence against the assumption of conditionally Gaussian TGARCH innovations. Estimation results documented in Table 2 indicate that the Gaussian copula never achieves the best fit. Instead, the Frank copula provides a better approximation for all series and the best fit across all copula specifications for 15 of the 18 TGARCH innovations series. Also the implied dependence parameters (Kendall's $\tau)$ are sizeable in general. While the Clayton copula also outperforms the Gaussian copula in 17 cases, it only provides best empirical approximations for three markets. Also the gains in approximation accuracy are less pronounced than for the Frank copula and the implied dependence parameters are generally smaller for the Clayton family. The Gumbel copula typically performs worse than the Gaussian and the implied dependence parameters are in most cases close to zero. For all Archimedean copulae, the best fitting rotations match with our remarks on tail dependence in Section 2.2. In order to assess the robustness of these findings, Figure 4 (Figure 5) shows distance measures between the Clayton (Frank) copula and $\operatorname{TGARCH}(1,1,1)$ innovations for four indices 7 In summary, we conclude that the (rotated) Frank copula obtains most accurate approximations of the autodependence structure that underlies consecutive TGARCH innovations.

${ }^{6}$ Most data is obtained from Yahoo!Finance (finance.yahoo.com) except for the DJIA 30 which has been drawn from the Federal Reserve Bank of St. Louis, Economic Research (http://research.stlouisfed.org/fred2/).

${ }^{7}$ The empirical findings are robust for all stock markets and the four indices have been selected for illustrative purposes only. The results for the Gumbel copula look similar to those for Clayton, except that rotations 2 and 4 provide smallest distance estimates that are, however, larger than respective statistics determined for the Gaussian copula. 


\subsection{Empirical forecast comparisons}

With the above results in mind, it is of particular interest if cVaR or cES forecast adjustments by means of the standardized Frank copula can improve upon the forecast accuracy of Gaussian-TGARCH models. In the following, we first comment on cVaR estimates and, second, provide a more detailed discussion of cES forecast diagnostics. Related to the ongoing discussion in the Basel framework it is worthwhile to mention that we put particular emphasis on results for the more conservative coverage levels $\alpha=0.01$ for the cVaR and the less conservative $\alpha \geq 0.025$ for the cES. If not stated explicitly our discussion of inferential results refers to the $5 \%$ significance level.

\subsection{1 $\quad \mathrm{cVaR}$ forecasting}

To see if the copula-adjusted cVaR forecasts outperform standard (Gaussian) forecasts in terms of unconditional VaR coverage, we first report empirical coverage estimates for alternative nominal levels $\alpha$ in Table 3. With regard to the Basel relevant nominal 1\% coverage level, we find a clear improvement of cVaR forecasts after adjustment by means of the standardized Frank copula. In specific, the Frank model obtains empirical coverage statistics closest to $1 \%$ for 17 out of 18 indices. This finding is robust for $\alpha=0.025$ with regard to 15 markets. Also for $\alpha=0.05$, the Frank cVaR empirical coverage is closest to the nominal level for the majority of markets. We conclude that adjusting $\mathrm{cVaR}$ forecasts by means of the Frank copula obtains most conservative risk measures for $\alpha=0.01$ and $\alpha=0.025$. In contrast, adjustments based on the standardized Clayton or Gumbel copula do not improve standard cVaR forecasts that are determined under the assumption of independent TGARCH innovations. Yet, for both conservative nominal levels, $\alpha=0.01$ and $\alpha=0.025$, all risk models appear overly liberal as implied by positive coverage errors, $\hat{\alpha}-\alpha>0$.

To test if the misspecification can be mitigated by employing the Frank copula, $p$-values for DQ-statistics are documented in Table 4. Overall, misspecification is commonly diagnosed for $\alpha=0.01$, while cVaR forecasts for $\alpha=0.05$ are characterized by incorrect unconditional or conditional coverage only for the DJIA 30 and S\&P 500. In most cases, rejection or acceptance decisions for the null hypothesis are identical across alternative copula based forecast adjustments. In a few cases test decisions differ across model families. For instance, distinct 
decisions are made for $\alpha=0.01$, where the null hypothesis for Frank adjusted forecasts is not rejected in three cases, compared with all other copula specifications. Likewise a uniquely not rejected model is found only for 3 or 2 indices for coverage levels of 0.01 and 0.025 , respectively. However, the $p$-values of the DQ-statistic indicate a predominance of the Frank model at the Basel-relevant $1 \%$ level and at the $2.5 \%$ level. The $p$-values obtained from the Frank model are higher than for other copulae for 11 indices at the $1 \%$ level and for 13 indices at the $2.5 \%$ level, thus indicating a better fit of the model. With regard to the more liberal 5\% coverage level, a dominance of the Frank model cannot be concluded.

Summarizing the performance of rival cVaR estimates, we note that the semiparametric forecast adjustments show potential to improve common risk measures. The diagnostic tests, however, indicate some remaining misspecification. As a consequence, the following discussion of cES estimates does not condition on pretest survival models but sheds light on the performance of all alternative approaches to risk assessment.

\subsection{2 cES forecasting}

As documented in Table 5, not surprisingly, Frank-adjusted cES forecasts are most conservative almost throughout, and especially so for the most conservative coverage level $\alpha=0.01$. In contrast, forecasts adjusted by means of the standardized Gumbel or Clayton copula are similar to respective statistics obtained from the common Gaussian model.

Applying the MF test for unbiasedness of the cES forecasts, we find that all models are correctly specified with respect to their cES forecasting performance. However, it must be noted that the exceedance residuals defined in (17) are only determined conditional on observing a cVaR hit first. Hence, the power of the test is likely to suffer from data scarcity.

As documented in Table 6 the mean cES forecast errors indicate that cES forecasts are insufficiently conservative, since all mean errors are negative and, thus, indicate an underestimation of the cES. Yet, in absolute values, the Frankadjusted forecasts show the smallest mean errors for $\alpha=0.025$ and $\alpha=0.05$ for 15 and 17 indices respectively. With regard to $\alpha=0.01$, the Frank performs best for only about half of the indices, however, it also generates the fewest hits (as seen in Table 3), and, hence, it is most prone to outliers. 
To compare forecast accuracy we report the copula-specific forecast MSE estimates relative to respective statistics from Gaussian forecasts in Table 7. For $\alpha=0.025$ and $\alpha=0.05$ the Frank-adjusted forecasts outperform Gaussian forecasts for 10 and 11 markets, respectively. However, results are generally inconclusive when comparing the accuracy of Frank-adjusted forecasts with the one from the Gaussian model, especially for smaller nominal coverage levels. For $\alpha=0.01$, the Frank achieves a lower MSE for only 7 indices, yet relative MSE statistics vary between $38.7 \%$ and $141.3 \%$. Recalling that the Frank adjusted cVaR forecasts obtain strictly fewer hits, this may partially be explained by the small sample size for which the cES is determined and, by implication, the strong effect of outliers. This is further supported by a decrease in the variation of relative MSE statistics documented for $\alpha=0.025$ and $\alpha=0.05$. In line with the previous results, the Clayton and Gumbel-adjusted forecasts are generally very similar in terms of forecast accuracy to those obtained from the Gaussian model. To test for significant differences in the forecast accuracy based on the MSE criterion, we applied the sign test. Our results show that the sign test cannot identify superior forecasts in terms of MSE prediction accuracy. However, as outlined in Section 3.2.2, the sign test may be potentially biased, although we account for a punishment effect if two forecasts differ in cVaR coverage rates. Therefore, respective test results must be viewed with caution.

So far, we have shown that forecast adjustments by the Frank copula generally provide the most conservative (cVaR and) cES forecasts. With regards to forecast accuracy, however, the results are mixed. To assess if copula forecast adjustments can help to reduce economic losses we next consider losses of a regulator who relies on the economic criterion in (19). We document regulator's losses in Table 8 for the three Archimedean copula forecast adjustments relative to the losses of the Gaussian forecast. Along the lines of the previous findings, the Clayton and Gumbel-adjusted forecasts typically feature regulator's losses close to, yet generally slightly larger than the ones obtained from the Gaussian model framework. The sign test indicates a superiority of the Gaussian forecasts compared with the Clayton- or Gumbel-adjusted forecasts.

In contrast to these copulae, the Frank adjusted forecasts reduce regulator's losses throughout. Since a forecasting error is here defined as a loss exceeding the copula-specific cES forecast, the Frank copula generally not only features the 
fewest losses but also, in case of a loss, the exceedance is the smallest. Hence, these gains for the Frank copula are substantial for all nominal coverage levels $\alpha$. For the larger nominal levels, economic gains are evident for all but three markets and losses are typically reduced by $10 \%$ to $20 \%$. The statistical significance of these results is supported by means of the sign test according to which the hypothesis of equal predictive ability between the copula-adjusted cES forecasts and the benchmark Gaussian cES forecast is strongly rejected. For the FrankGaussian comparison this rejection is uniquely in favor of the Frank and most frequently observed for $\alpha=0.025$ and $\alpha=0.05$. This can be partially credited to the limited sample size for $\alpha=0.01$. For a regulator with a loss function as specified in (19), we conclude that losses may be reduced significantly when adjusting cES forecasts by means of the standardized (rotated) Frank copula.

\section{Conclusions}

Based on the class of standardized copula distributions introduced by Lee \& Long (2009) we propose a semiparametric framework to capture higher order dependence structures in standardized threshold GARCH innovations. The model framework is motivated from empirical autodependence patterns as identified within a large cross section of stock market processes. Although it relies on unconditionally (marginally) Gaussian innovations, the proposed model allows for the ex-ante determination of nontrivial return quantiles, and, thus, is of potential interest for purposes of risk management. We apply the new model to derive conditional estimates of both value-at-risk and expected shortfall. As it turns out, in particular mimicking empirical dependence structures by means of the standardized Frank copula leads to an improvement of standard dynamic risk measures that have been previously build under the assumption of bivariate Gaussian innovation tuples. Frank-adjusted forecasts for the conditional VaR and ES are generally more conservative and may reduce extreme losses to economic agents

significantly. In particular, when taking the perspective of a regulating authority the Frank based cES adjustments are valuable in economic terms.

With regard to future research we notice that the set of applied copula distributions in this work has been limited to the Clayton, Gumbel and Frank families. Similarly, marginal distributions have been presumed to be Gaussian. 
Hence, further improvements in forecast adjustments could be expected from increasing the set of copulae or presuming heavy-tailed marginal distributions. Moreover, instead of matching a discrete set of parameterized copula distributions to the empirical counterparts, a finer tuning of the parameters that characterize the best-fitting might be obtained by moment based estimation techniques. Furthermore, we throughout tried to approximate the entire conditional distribution of innovations underlying GARCH processes. With regard to conditional risk assessment, one may opt to concentrate more on conditional tail characteristics. In this respect it is worthwhile to mention that the considered class of standardized bivariate copula allows to focus on tail dependence in a semiparametric and flexible manner. 


\section{References}

Angelidis, T. \& Degiannakis, S. (2007). Backtesting VaR models: a two-stage procedure. Journal of Risk Model Validation, 1(2), 27-48.

Basel Committee on Banking Supervision (2005). Amendment to the Capital Accord to incorporate market risk.

Basel Committee on Banking Supervision (2012). Fundamental review of the trading book.

Bollerslev, T. (1986). Generalized autoregressive conditional heteroskedasticity. Journal of Econometrics, 31(4), 307-327.

Bollerslev, T. (1990). Modelling the coherence in short-run nominal exchange rates: A multivariate generalized $\mathrm{ARCH}$ model. The Review of Economics and Statistics, 72(3), 498-505.

Cai, Z. (2001). Weighted Nadaraya-Watson regression estimation. Statistics \&6 Probability Letters, 51(3), 307-318.

Cai, Z. (2002). Regression quantiles for time series. Econometric Theory, 18, 169-192.

Cai, Z. \& Wang, X. (2008). Nonparametric estimation of conditional VaR and expected shortfall. Journal of Econometrics, 147(1), 120-130.

Cherubini, U., Luciano, E., \& Vecchiato, W. (2004). Copula methods in finance. Hoboken N.J.: John Wiley \& Sons.

Diebold, F. X. \& Mariano, R. S. (1995). Comparing predictive accuracy. Journal of Business 6 Economic Statistics, 13(3), 134-144.

Diks, C. G. H., Panchenko, V., \& van Dijk, D. J. C. (2011). Likelihood-based scoring rules for comparing density forecasts in tails. Journal of Econometrics, $163(2), 215-230$.

Engle, R. F. (1982). Autoregressive conditional heteroscedasticity with estimates of the variance of United Kingdom inflation. Econometrica, 50(4), 987-1007. 
Engle, R. F. \& Manganelli, S. (2004). CAViaR: Conditional autoregressive value at risk by regression quantiles. Journal of Business 8 Economic Statistics, 22(4), 367-381.

Glosten, L. R., Jagannathan, R., \& Runkle, D. E. (1993). On the relation between the expected value and the volatility of the nominal excess return on stocks. The Journal of Finance, 48(5), 1779-1801.

Granger, C. W. J. \& Pesaran, M. H. (2000). Economic and statistical measures of forecast accuracy. Journal of Forecasting, 19(7), 537-560.

Hall, P., Wolff, R. C., \& Yao, Q. (1999). Methods for estimating a conditional distribution function. Journal of the American Statistical Association, 94(445), 154-163.

Herwartz, H. (2012). On the predictive content of autoregression residuals: a semiparametric, copula-based approach to time series prediction. Journal of Forecasting, in press.

Lee, T.-H. \& Long, X. (2009). Copula-based multivariate GARCH model with uncorrelated dependent errors. Journal of Econometrics, 150(2), 207-218.

McNeil, A. J. \& Frey, R. (2000). Estimation of tail-related risk measures for heteroscedastic financial time series: an extreme value approach. Journal of Empirical Finance, 7(3-4), 271-300.

Nelsen, R. B. (2006). An introduction to copulas. New York: Springer, 2 edition.

Sarma, M., Thomas, S., \& Shah, A. (2003). Selection of value-at-risk models. Journal of Forecasting, 22, 337-358.

Yu, K. \& Jones, M. (1998). Local linear quantile regression. Journal of the American Statistical Association, 93(441), 228-237.

Zhu, D. \& Galbraith, J. W. (2011). Modeling and forecasting expected shortfall with the generalized asymmetric Student-t and asymmetric exponential power distributions. Journal of Empirical Finance, 18, 765-778. 


\section{A Matching and density estimation}

\section{A.1 Copula matching}

In order to determine the closest approximation of the estimated model innovations by means of a copula distribution, we contrast a discretized empirical distribution of the estimated innovations against simulated copula counterparts by means of a common goodness-of-fit statistic. Next, we briefly provide the employed discretization of bivariate distributions and then outline how particular copula models are selected in a data driven manner.

\section{A.1.1 Estimation of discretized frequencies}

While an arbitrarily precise approximation of the continuous distribution of simulated copula data could be obtained by means of simulations, the restricted length of available financial time series data calls for employing a rough grid for discretization (Herwartz, 2012). With about 2200 observations for most empirical time series used in this study (see Section 4.1) we employ a bivariate grid consisting of $8 \times 8=64$ cells.

In order to obtain a reliable approximation of the distribution of selected copulae, $R=10000$ samples of length $S=2000$ are drawn from each parameterized (rotated) copula obtaining a set of bivariate random variables $\left(\mathbf{v}_{1}^{r}, \mathbf{v}_{2}^{r}, \ldots, \mathbf{v}_{S}^{r}\right)$, $(r=1, \ldots, R)$, where $\mathbf{v}_{s}^{r}=\Sigma^{-\frac{1}{2}} \mathbf{w}_{s}^{r}$. The joint density of each sample $\left\{\mathbf{v}_{s}^{r}\right\}_{s=1}^{S}$ is then approximated by means of the following empirical frequencies:

$$
h_{r}(m, n)=\frac{1}{S} \sum_{s=1}^{S} I\left(\left(a_{m} \leq v_{1 s}^{r}<a_{m+1}\right)\left(a_{n} \leq v_{2 s}^{r}<a_{n+1}\right)\right) \text {, }
$$

where $a_{m}, a_{n}$, with $m, n=1, \ldots, 8$, are taken from the grid vector $a=(-1000,-1.5,-1.0,-0.5,0,0.5,1.0,1.5,1000)^{\prime}$. An estimate of the discretized distribution for each copula is then

$$
H_{(m, n)}=\frac{1}{R} \sum_{r=1}^{R} h_{r}(m, n)
$$

where $H_{(m, n)}$ is the $m, n$ element in the discretized distribution $H$. By this procedure a set comprising the Gaussian, 72 Clayton and Gumbel and 36 Frank 
distributions is obtained and denoted as $H^{\Phi}, H_{j}^{C}, H_{j}^{G}, j=1, \ldots, 72$ and $H_{j}^{F}$, $j=1, \ldots, 36$, respectively.

The empirical counterpart of the model specific distribution $H_{(m, n)}$ reads as

$$
\hat{h}_{(m, n)}=\frac{1}{T-1} \sum_{t=1}^{T-1} I\left(\left(a_{m} \leq \hat{\xi}_{t}<a_{m+1}\right)\left(a_{n} \leq \hat{\xi}_{t+1}<a_{n+1}\right)\right) .
$$

where $\hat{\xi}_{t}$ are the standardized TGARCH innovations, $\hat{\xi}_{t}=r_{t} / \hat{\sigma}_{t}$.

\section{A.1.2 Copula selection}

The selection of the best copula from each family is implemented according to a distance measure between the approximated empirical distribution and the simulated counterparts. The distance function follows Herwartz (2012) and reads as

$$
D_{j}^{\bullet}=\sum_{m} \sum_{n} \frac{\left(H_{j,(m, n)}^{\bullet}-\hat{h}_{(m, n)}\right)^{2}}{H_{j,(m, n)}^{\bullet}}
$$

To find target distributions $H_{j^{*}}^{\bullet}$, this distance measure is minimized for each family of copula distributions, i.e. $j^{*}=\arg \min _{j} D^{\bullet, j}$ for $\bullet \in\{C, F, G\}$. Owing to the implied independence the Gaussian model is unique and always evaluated for the purpose of benchmarking. These target distributions $j^{*}$ for each of the three Archimedean copula families are then employed for $\mathrm{cVaR}$ and $\mathrm{cES}$ forecast adjustments based on the previously estimated TGARCH processes described in Section 3.1.

\section{A.2 Copula density and quantile estimation}

As the forecasting approach outlined in Section 3.1 requires the inversion of the model innovations' cCDF, we employ a Nadaraya-Watson (NW) estimator 8 to approximate the distribution of $\xi_{t+1}$ given $\xi_{t}$. The cCDF estimate is obtained

8 Hall et al. (1999) develop a weighted Nadaraya-Watson (WNW) estimator that preserves the property of the NW estimator to always obtain proper CDFs and also inherits the unbiasedness property of the local linear estimator proposed by Yu \& Jones (1998). See also Cai (2001) and Cai (2002). In this application, the weights assigned by the WNW estimator are, however, found to be identical for all realizations. Hence, the WNW cannot be distinguished in statistical terms from the NW estimator here. 
from the respective simulated innovations $\mathbf{v}_{s}^{r}, s=1, \ldots, S$ along grid vectors $x=$ $y=[-4.0,-3.9, \ldots, 4]$ where $x$ and $y$ represent the conditioning and dependent variable respectively. The cCDF estimate at grid point $y$ given $x$ reads as

$$
\hat{F}(y \mid x)=\frac{\sum_{s=1}^{S} K_{h}\left(x-v_{1, s}\right) I\left(v_{2, s} \leq y\right)}{\sum_{s=1}^{S} K_{h}\left(x-v_{1, s}\right)},
$$

where $K_{h}()=.\frac{1}{h} K(\dot{\bar{h}})$ is the kernel function and $h>0$ is the bandwidth parameter 9

From this discretized estimate of the conditional CDF, cVaR and cES estimates can be obtained. In the general form, the estimate for the conditional quantile is

$$
\hat{q}_{\alpha}(y \mid x)=-\hat{F}^{-1}[\alpha \mid x]
$$

where the approximation is along the grid vector $x=(-4.0,-3.9, \ldots, 4.0)^{\prime}$ and linear interpolation is applied to determine $\hat{q}_{\alpha}$. With $\hat{q}_{\alpha}$ at hand, the cES can subsequently be estimated by means of the plugging-in method Cai \& Wang (2008) as:

$$
\widehat{\operatorname{cES}}_{\alpha}(y \mid x)=-\frac{1}{\alpha} \int_{-\infty}^{\hat{q}_{\alpha}(y \mid x)} y \hat{f}(y \mid x) d y .
$$

As the estimated conditional CDF is discrete, an approximation of (27) is

$$
\widehat{\operatorname{cES}}_{\alpha}(y \mid x)=-\frac{1}{\alpha} \sum_{y_{i} \leq \hat{q}_{\alpha}(y \mid x)}\left(y_{i}-\frac{y_{i}-y_{i-1}}{2}\right)\left[\hat{F}\left(y_{i} \mid x\right)-\hat{F}\left(y_{i-1} \mid x\right)\right]
$$

where $y_{i}$ is the $i$ 'th element of the grid vector $y=(-4.0,-3.9, \ldots, 4.0)^{\prime}$ and $\frac{y_{i}-y_{i-1}}{2}$ accounts for the discreteness of estimates.

\footnotetext{
${ }^{9}$ In this study, the Gaussian kernel defined as $K()=.\frac{1}{\sqrt{2 \pi}} \exp \left(-\frac{1}{2}\left(x-x_{s}\right)^{2}\right)$ and a bandwidth $h=1.06(S-1)^{-0.2}$ are used throughout and yield satisfactory results. An optimal bandwidth rule for conditional quantile estimation can be found in Cai (2002).
} 


\section{B Figures}
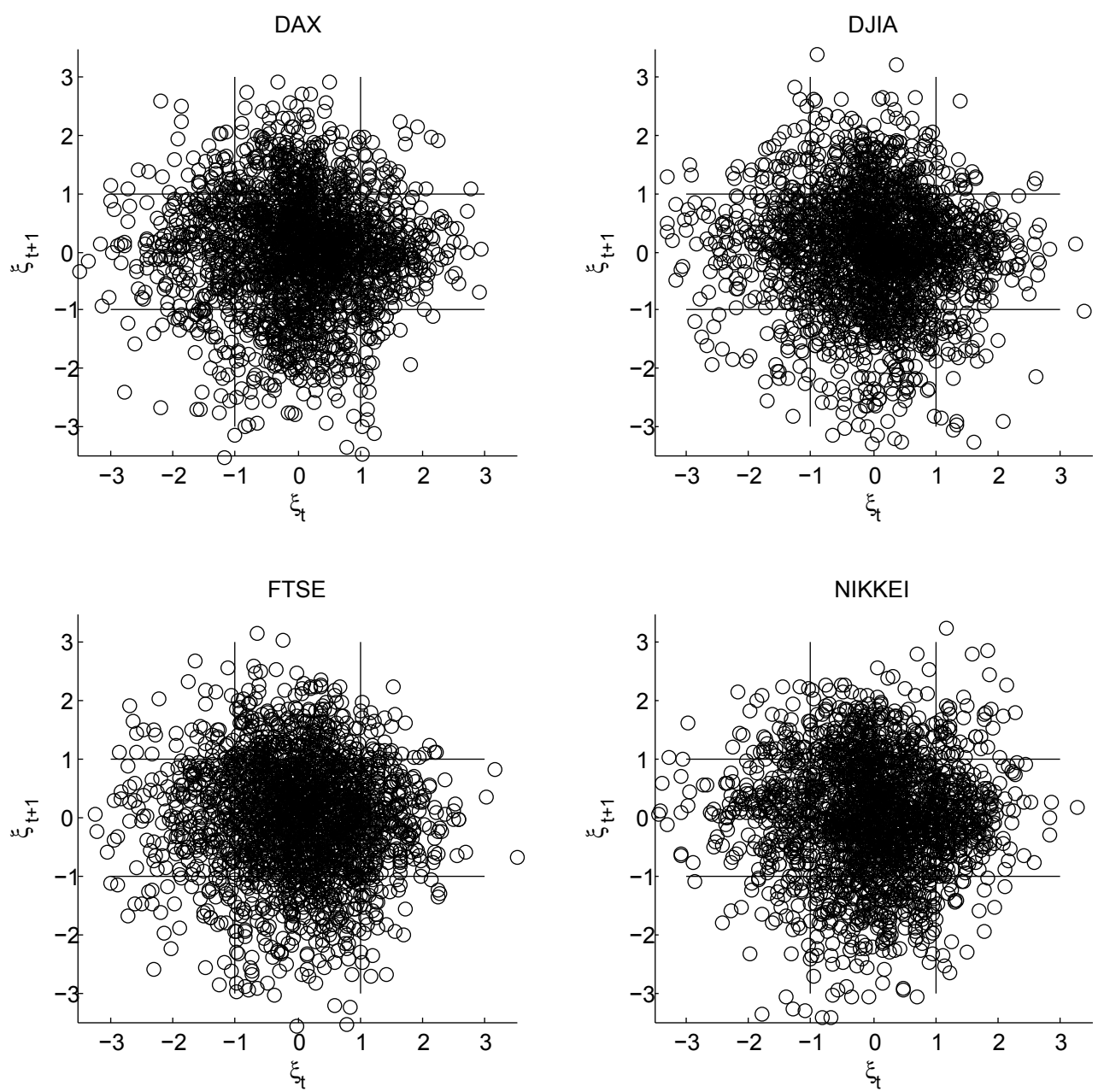

Figure 1: Standardized TGARCH $(1,1,1)$ residuals $\left(\xi_{t}\right.$ vs. $\left.\xi_{t+1}\right)$ for four indices from QML estimation. Solid lines indicate the absolute unit levels. 

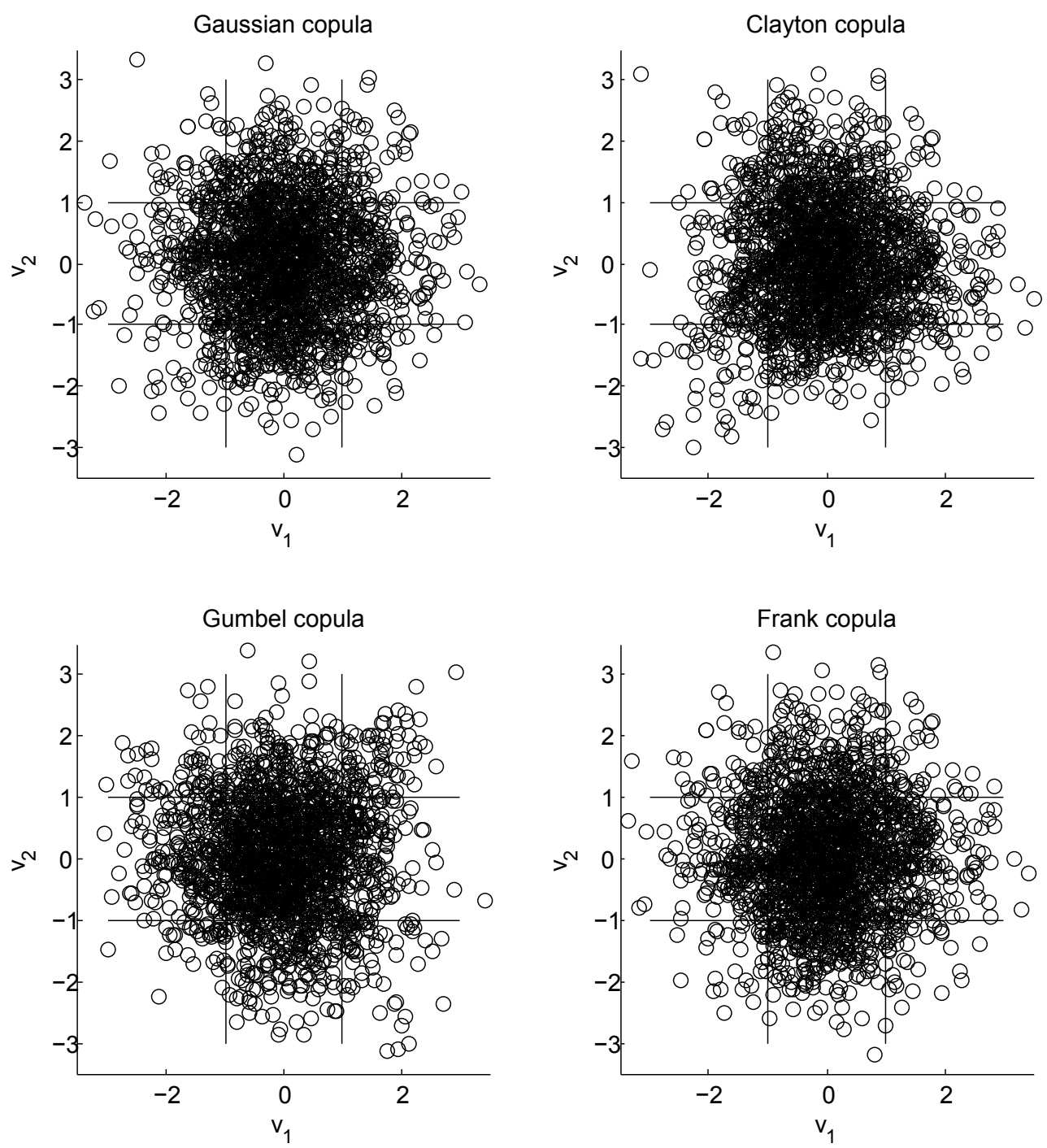

Figure 2: Scatter plots for samples from standardized Gaussian and Archimedean copulae with $\tau=0.250$ prior to standardization. For further notes see Figure 1 

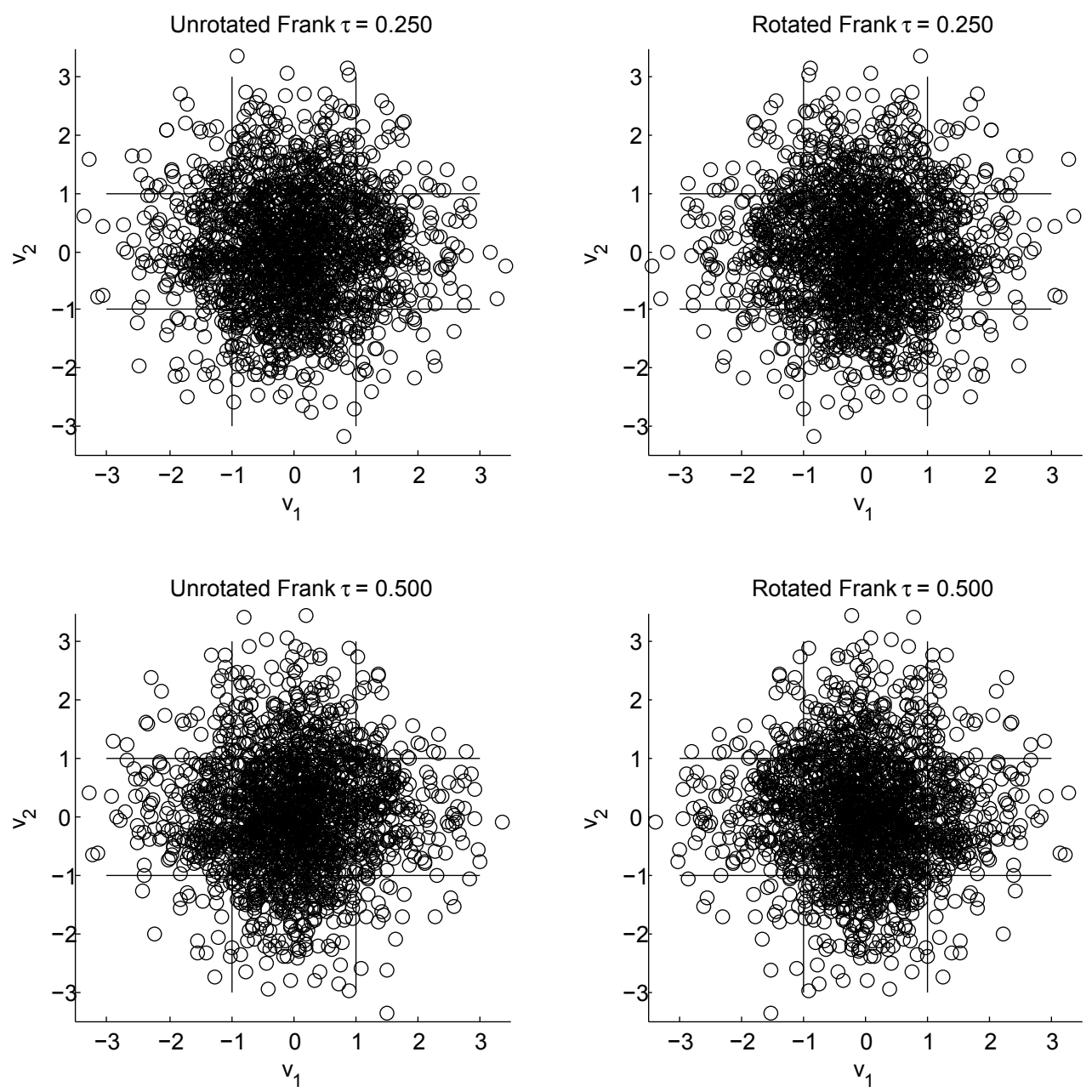

Figure 3: Scatter plots for samples from standardized (rotated) Frank copula with $\tau=0.25,0.50$ prior to standardization. For further notes see Figure 1 . 

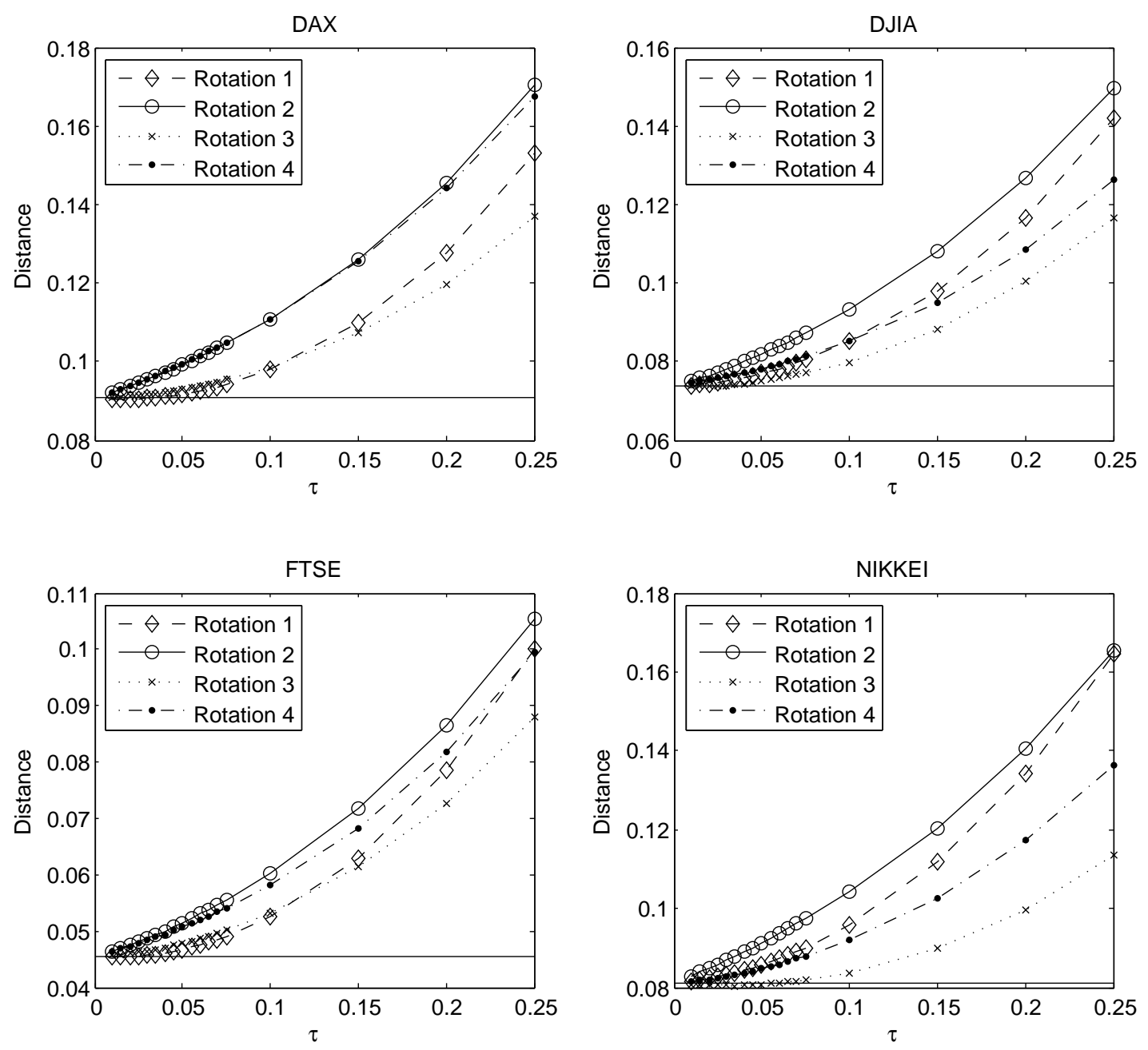

Figure 4: Distance between samples from standardized (rotated) Clayton copulae and TGARCH $(1,1,1)$ residuals derived from four stock indices (solid horizontal line indicates distance of the Gaussian). The $x$-axis corresponds to the dependence parameter $\tau$ prior to copula standardization. 

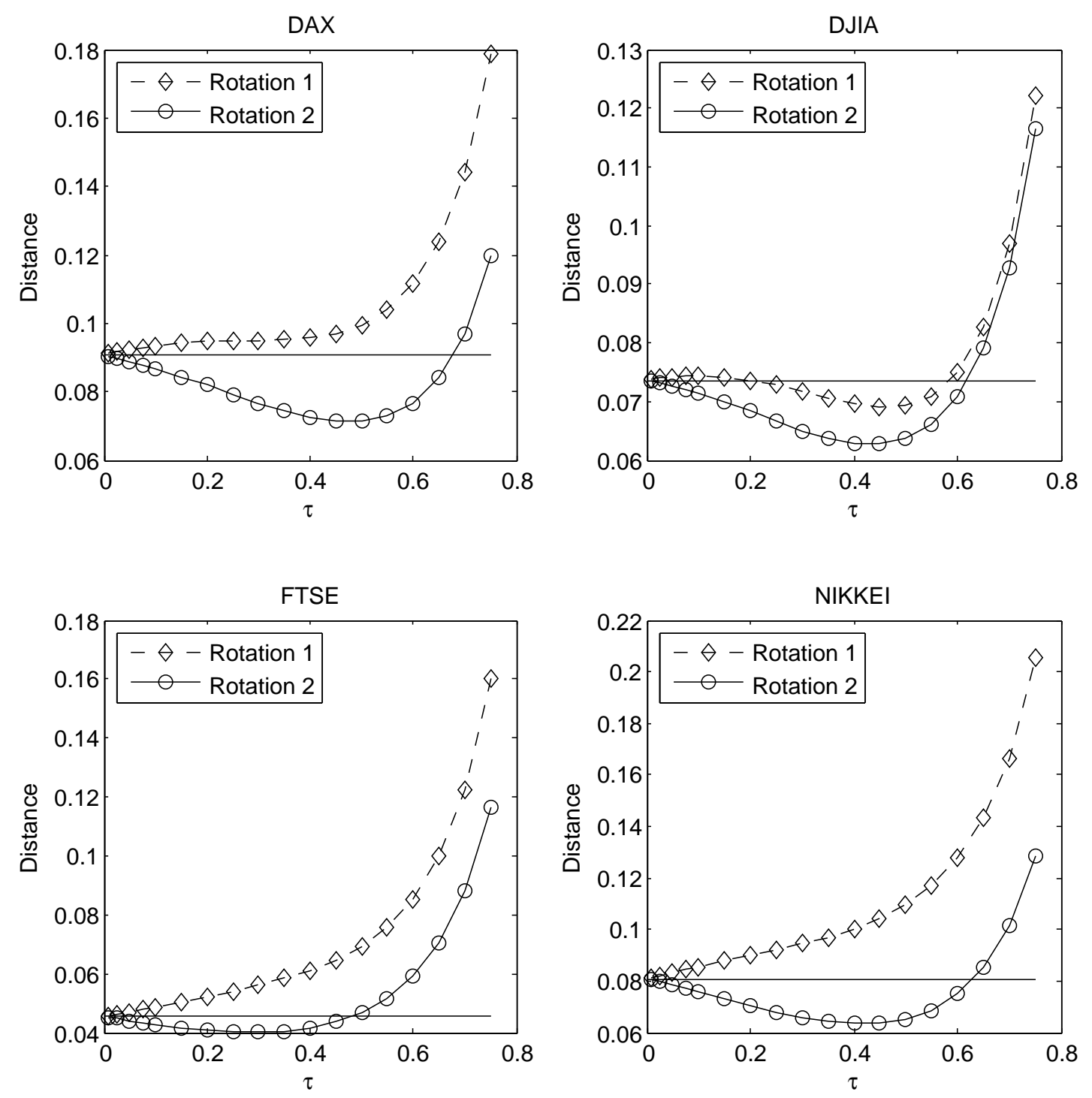

Figure 5: Distance between samples from standardized (rotated) Frank copulae and TGARCH $(1,1,1)$ residuals. For further notes see Figure 4 . 


\section{Tables}

Table 1: Data set and TGARCH parameter estimates

\begin{tabular}{llrrrrrr}
\hline \hline Index & Country & $\mathrm{T}$ & $\gamma_{0}$ & $\gamma_{1}$ & $\gamma_{1}^{-}$ & $\beta_{1}$ & Log-Lik \\
\hline BSE Sensex 30 & India & 2229 & $7.14 \mathrm{E}-06$ & .050 & .144 & .858 & 6305.17 \\
CAC 40 & France & 2308 & $2.48 \mathrm{E}-06$ & -.024 & .183 & .919 & 6992.90 \\
DAX 30 & Germany & 2300 & $2.84 \mathrm{E}-06$ & $-.015^{\dagger}$ & .163 & .918 & 6929.51 \\
DJIA 30 & USA & 2266 & $1.61 \mathrm{E}-06$ & -.028 & .174 & .924 & 7443.02 \\
Euro Stoxx 50 & Eurozone & 2317 & $2.05 \mathrm{E}-06$ & -.028 & .182 & .926 & 7007.46 \\
FTSE 100 & UK & 2274 & $1.43 \mathrm{E}-06$ & $-.010^{\dagger}$ & .156 & .920 & 7335.50 \\
IBEX 35 & Spain & 2287 & $2.52 \mathrm{E}-06$ & $-.007^{\dagger}$ & .169 & .909 & 6941.38 \\
IBRX 50 & Brazil & 2215 & $8.48 \mathrm{E}-06$ & $.019^{\dagger}$ & .102 & .901 & 6034.37 \\
IPC 35 & Mexico & 2267 & $3.83 \mathrm{E}-06$ & $.009^{\dagger}$ & .148 & .897 & 6879.95 \\
JKSE & Indonesia & 2202 & $1.45 \mathrm{E}-05$ & .044 & .192 & .800 & 6405.46 \\
KOSPI & South Korea & 2230 & $6.79 \mathrm{E}-06$ & $-.005^{\dagger}$ & .173 & .883 & 6542.92 \\
MIB 40 & Italy & 2313 & $1.41 \mathrm{E}-06$ & $-.005^{\dagger}$ & .132 & .927 & 7087.52 \\
Nasdaq 100 & USA & 2267 & $3.32 \mathrm{E}-06$ & $-.009^{\dagger}$ & .138 & .922 & 6741.03 \\
Nikkei 225 & Japan & 2207 & $5.83 \mathrm{E}-06$ & .026 & .151 & .870 & 6454.65 \\
RTSI 50 & Russia & 2287 & $1.45 \mathrm{E}-05$ & .047 & .114 & .861 & 5899.33 \\
S\&P 500 & USA & 2267 & $1.54 \mathrm{E}-06$ & -.029 & .161 & .934 & 7294.96 \\
S\&P TSX & Canada & 2234 & $1.88 \mathrm{E}-06$ & $.009^{\dagger}$ & .115 & .913 & 7285.75 \\
SSEC & China & 2274 & $2.60 \mathrm{E}-06$ & .042 & .015 & .942 & 6267.05 \\
\hline \hline
\end{tabular}

Own calculations using EViews (version 5.1) and the ML-ARCH (Marquardt) algorithm Parameters indicated with ${ }^{\dagger}$ are zero with $5 \%$ significance. 


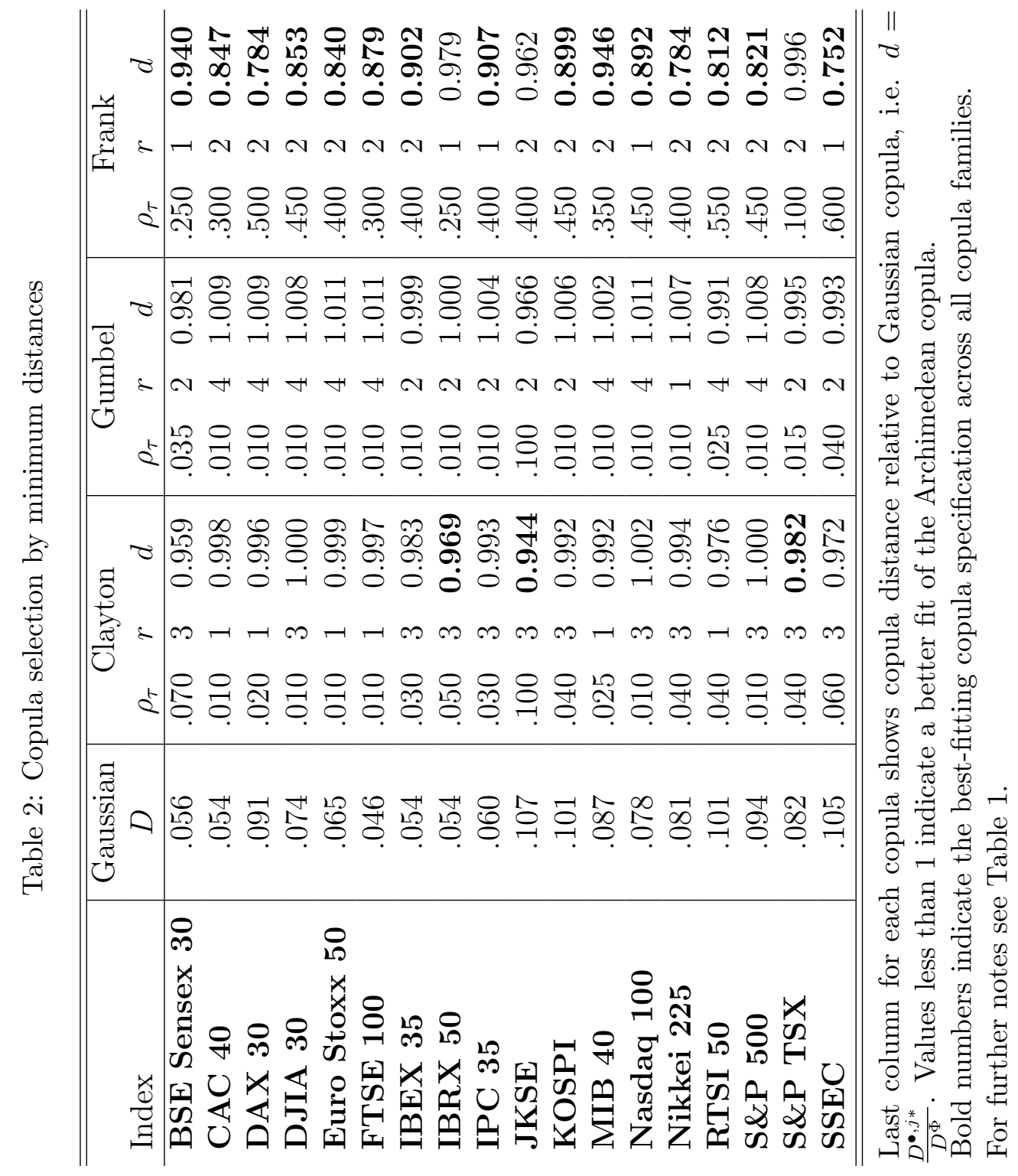




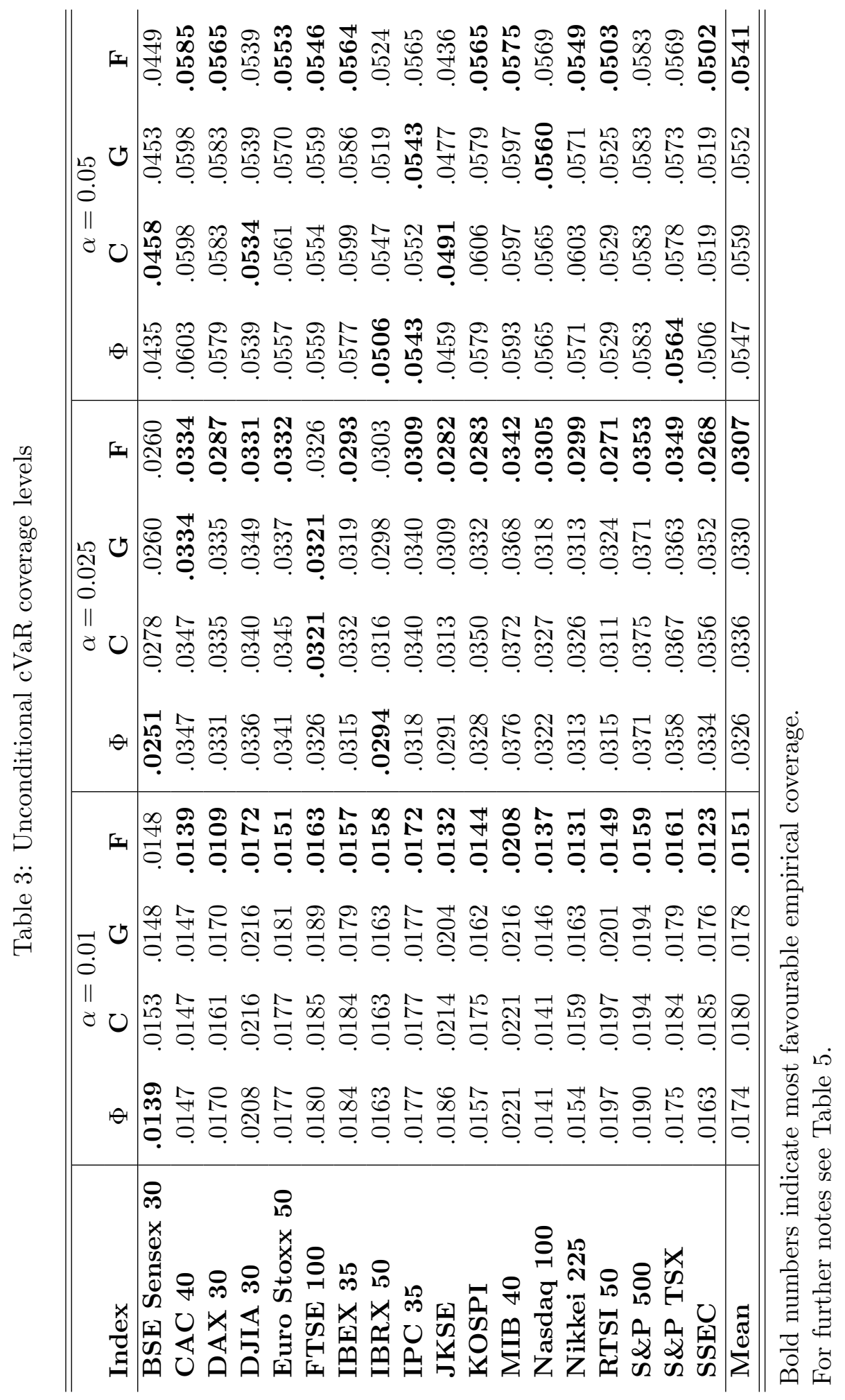




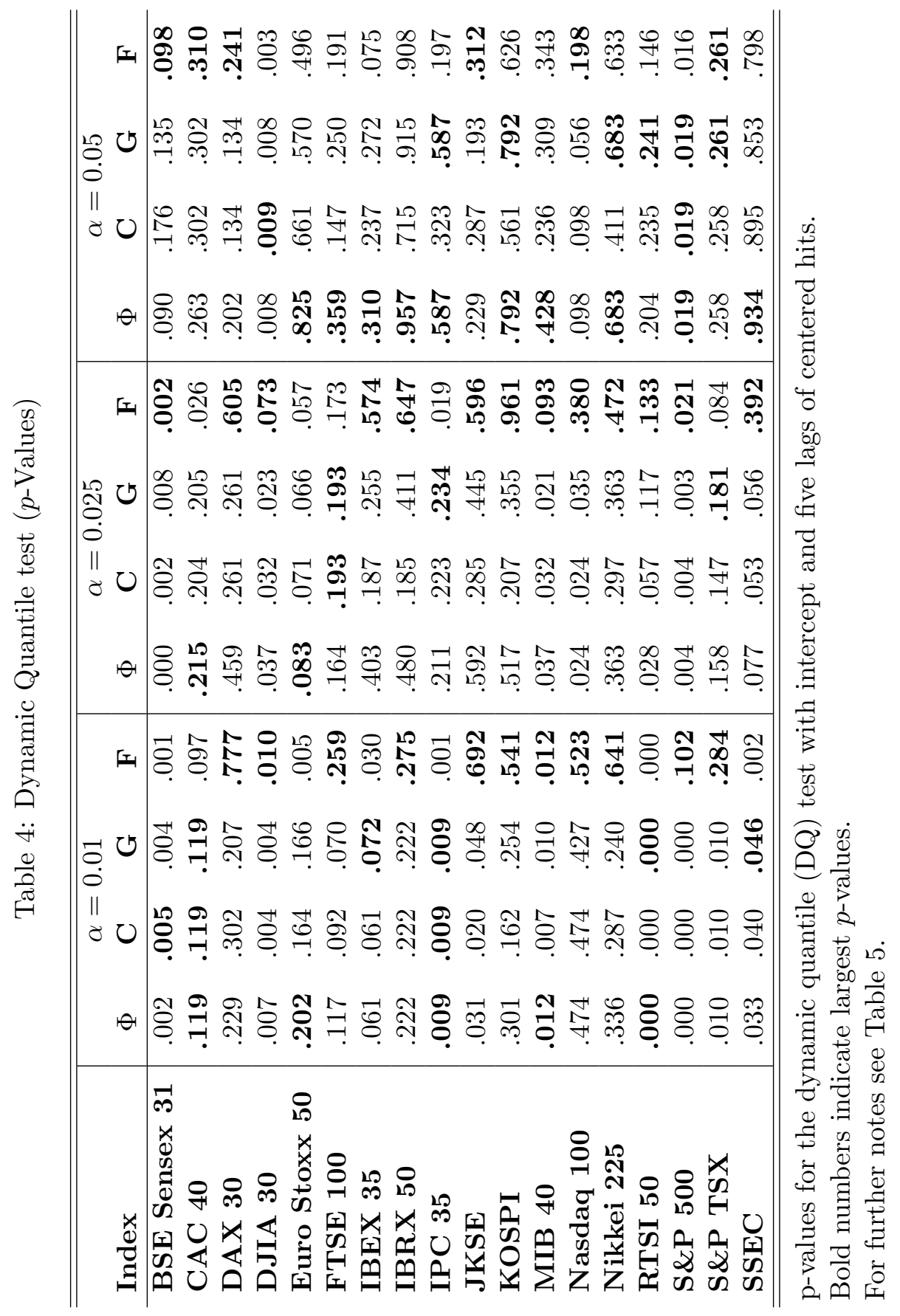




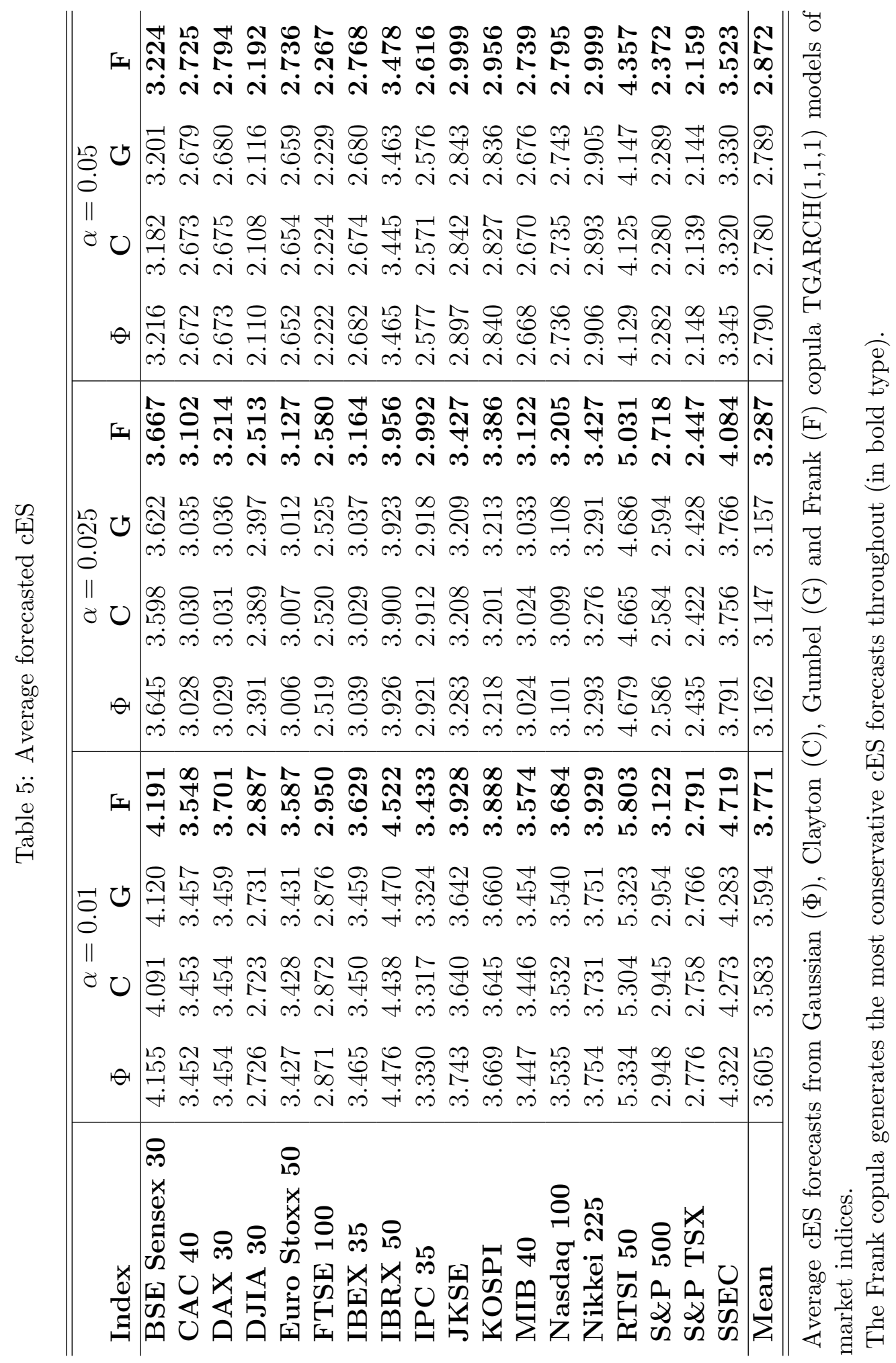




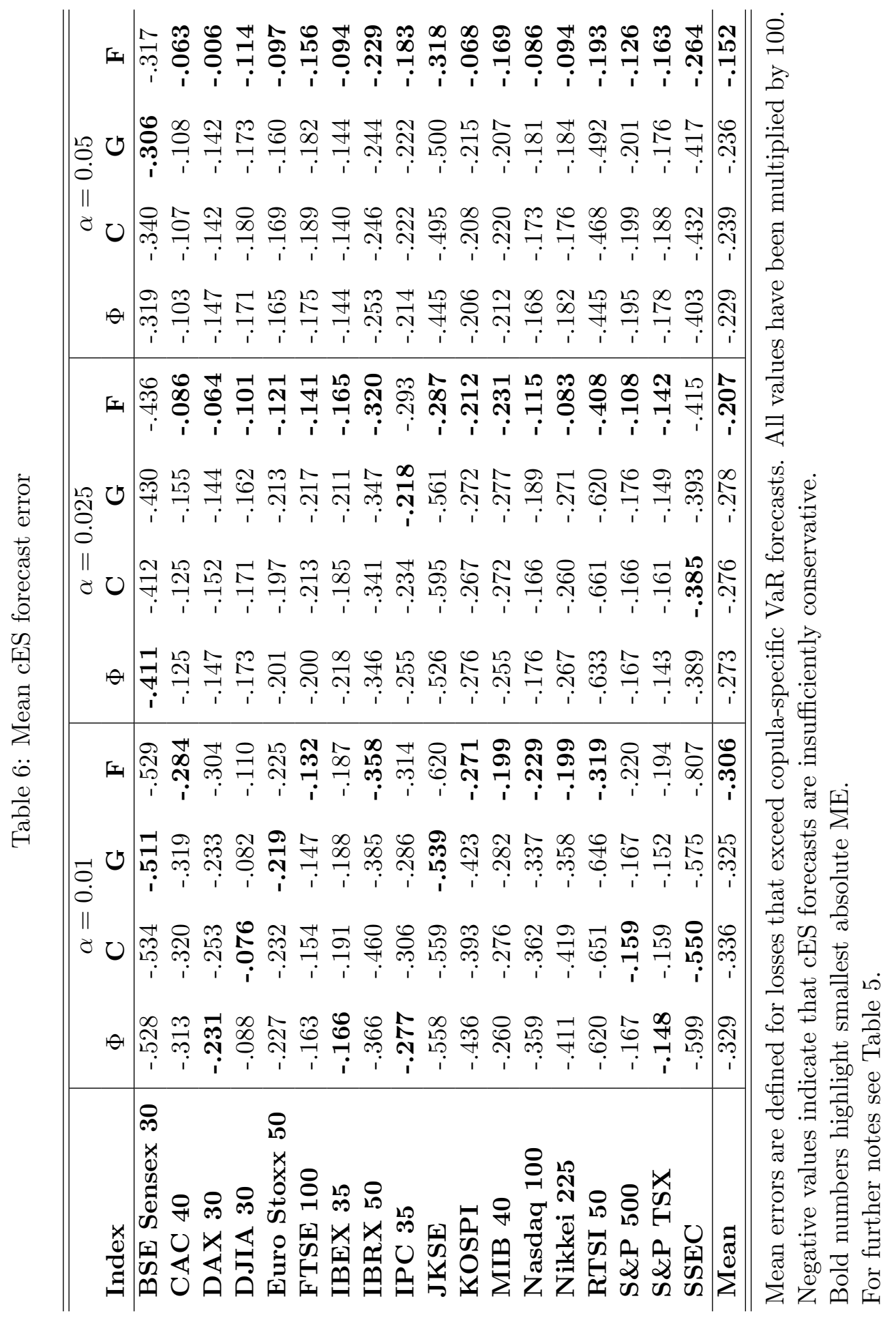




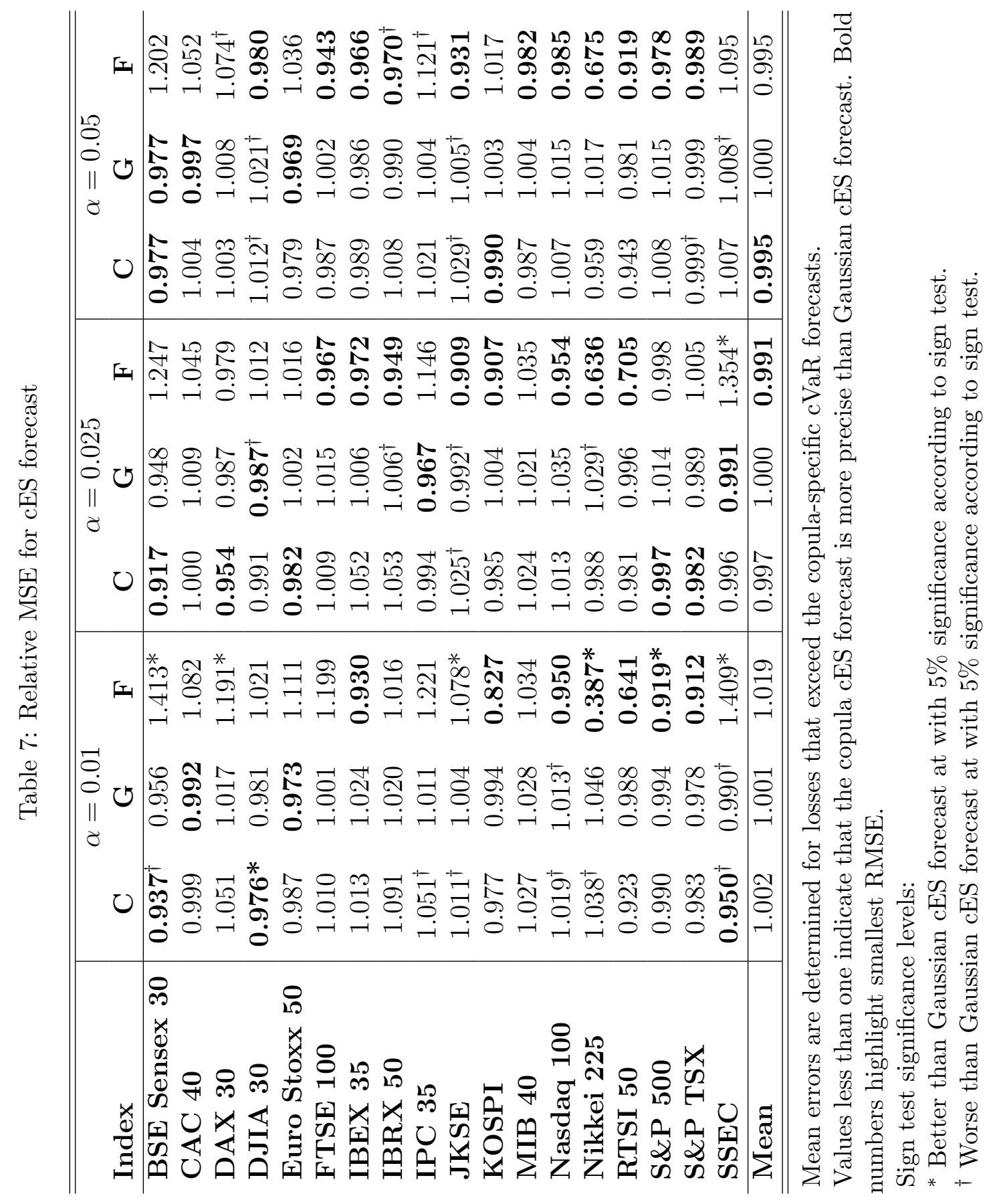




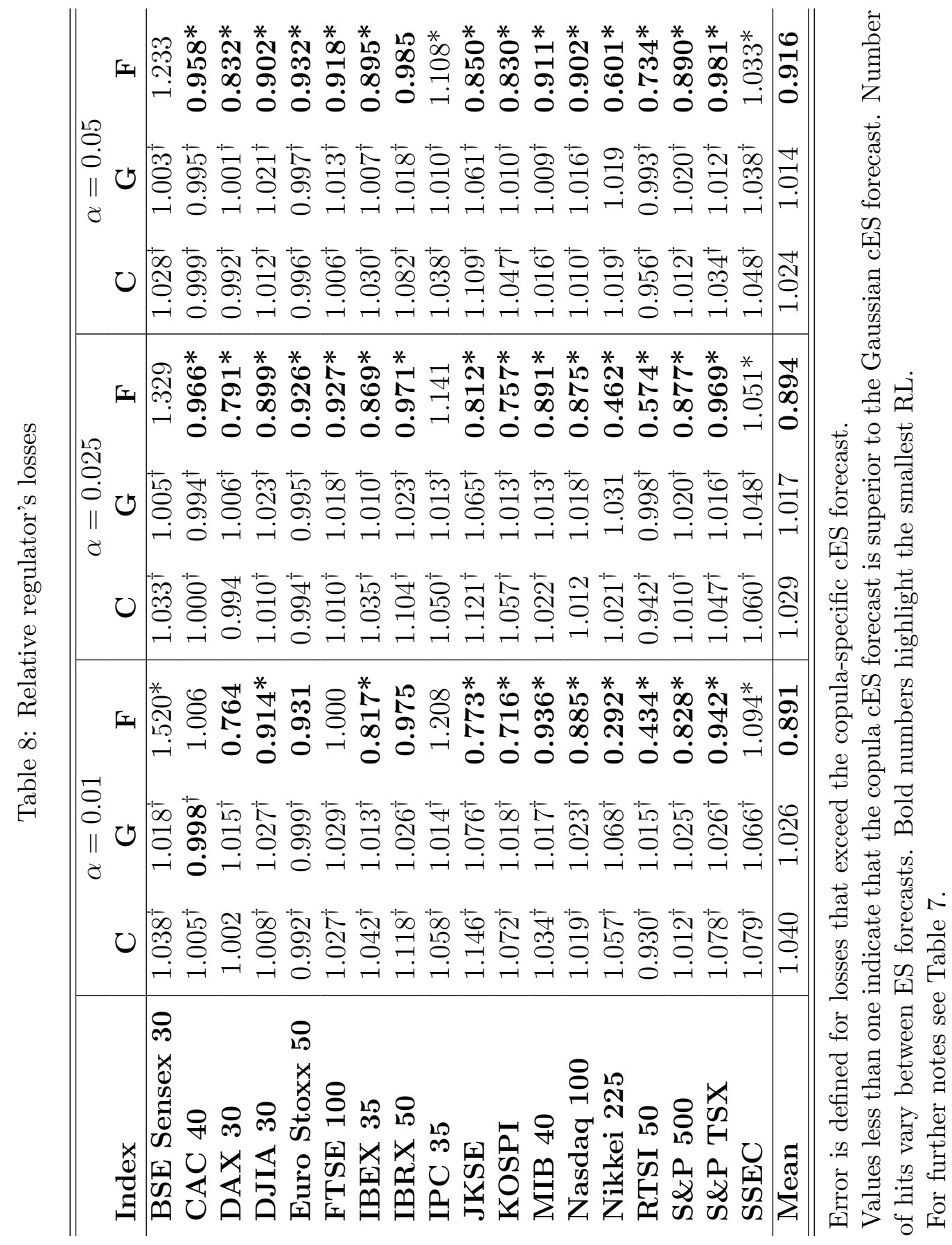

